\title{
Combating Leishmaniasis through Awareness Campaigning: A Mathematical Study on Media Efficiency
}

\author{
Dibyendu Biswas, Abhirup Datta, Priti Kumar Roy* \\ Centre for Mathematical Biology and Ecology \\ Department of Mathematics, Jadavpur University Kolkata - 700032, India \\ *Corresponding author: pritiju@gmail.com
}

(Received July 28, 2016; Accepted August 27, 2016)

\begin{abstract}
Leishmaniasis is a complex vector-borne disease that is originated by protozoa of the genus Leishmania. The disease is transmitted to human or animals through bites of infected female Phlebotominae sand-fly. Awareness campaign plays a significant role to restrict the spreading of disease cutaneous leishmaniasis. Here, we have considered a mathematical model of cutaneous leishmaniasis (CL) consisting susceptible and infected population of human and vector. Increase in growth of aware population impulsively leading to reduction of incompetence for environmental factors. Our analytical and numerical results reveal that circulating of the campaigning periodically minimizes the disease prevalence. The efficiency of the awareness programme contributes a better regime on the system moving towards healthy and hygienic environment.
\end{abstract}

Keywords- Vector-borne disease, cutaneous leishmaniasis (CL), awareness programme, impulsive approach.

\section{Introduction}

Leishmaniasis is considered as one of the seventeen most significant neglected tropical diseases (NTDs) globally according to World Health Organization (WHO, 2009). It is vectored by parasites of the genus Leishmania that transinfect the human and animals through bites of infected female sand-flies during blood meal of the sub-family named Phlebotominae (Kendrick, 1999). The disease has an occurrence of two million cases and is approximated over 50,000 deaths per year (Langer et al. 2012). After the incubation of ten days to two years period, some indications of fever, diarrhea, loss of body weight, lymphadenopathy, hepatomegaly and splenomegaly are found. The disease starts as an erythematous papule at the site of the sand-fly bite on outer part of the body. The papule turns into a nodule marked with distinct edge (Bathena, 2009; Reithinger et al. 2007). But there is still deficiency in the consideration about the parasite metabolism and progression of disease.

Transmission dynamics for cutaneous leishmaniasis has been studied under the framework of different mathematical models. Rabinovich and Feliciangeli (2004) formulated a mathematical model with consideration of probabilistic approach for number of infective bites by sand-flies. Lysenko and Beljaev (1987) proposed another mathematical model on cutaneous leishmaniasis for transmission of parasites. Chaves and Hernandez (2004) suggested a mathematical model involving vector, host and reservoir population for American cutaneous leishmaniasis. Marcos and Moreira (2007) developed the mathematical model of immune response in cutaneous leishmaniasis. Recently, Biswas et al. (2016) showed that delay can have significant impact in spreading the infection during the macrophagic phase of human stage. Das et al. (2007) studied the disease transmission of American Cutaneous Leishmaniasis with delay effect. Bacaer and Guernaoui (2006) proposed the mathematical model for the seasonality of the vector population. Simultaneously restraining mechanisms of the disease have already been explored in mathematical 
International Journal of Mathematical, Engineering and Management Sciences

Vol. 1, No. 3, 139-149, 2016

https://dx.doi.org/10.33889/IJMEMS.2016.1.3-015

as well as clinical points of view. But till date it is less explored that, awareness campaign may play as one of the foremost disease controlling method for cutaneous leishmaniasis. On that basis, we have taken the contemporary thinking of awareness campaign into our mathematical framework for model formulation.

Now, to control the disease, we have introduced the concept of awareness programs driven by social media into our research. Such, behavioral design can be developed in the mass population towards the disease transmission through awareness campaigns. It may create responsiveness amongst host (human) population. Proper methodical awareness packages can be one of the most proficient mechanisms for lowering the spreading of infection. It supports to shorten the incident of disease transmission. Most of these social campaigns through mass-media basically consider the awareness schedule which will reduce the contact between healthy and infected population by inducing knowledge sharing about counter measures to contain disease. As soon as the awareness programs are distributed amongst the population, the individual can react towards the scheme. The media campaigns are essentially run upon the information or knowledge mainly about the transmission of disease. Thus the campaigns hasten the actions, which can condense the probabilities of being infected. The influence of awareness campaigns, scattered by social mass media has been analyzed under mathematical understanding in many research articles (Nyabadza et al., 2010; Funk et al., 2009; Liu and Cui, 2008; Misra et al., 2011). The class of susceptible population, separated by awareness programs forms a new section in the population, called aware population. It is considered that the aware class is assumed to be protected from the infected class with the help of media generated campaigns. Due to some social or environmental factors, aware population may lose its awareness in future time (Misra et al., 2011).

In this research article, we have extended the work of Bacaer and Guernaoui (2006) incorporating awareness campaign to control the disease cutaneous leishmaniasis under mathematical context. We have considered the geographic area, where there is presence of social media. The major target of our work is to control the disease with the help of awareness campaign impulsively and to find out the maximum effectiveness of campaigning.

\section{Model Formulation with Suitable Assumptions}

We have considered the disease transmission is occurred between two different population, host (human) population and vector (sand-fly) population. The host population is divided into two groups, viz. susceptible individuals $S_{H}(t)$ and infected individuals $I_{H}(t)$. We do not consider the reservoir class because our focus is to make avoid contact between host and the vector. We have assumed reservoir population is in steady state. The recruitment rates of human and sand-fly population are taken as $\lambda_{H}$ and $\lambda_{V}$ respectively, which are considered as constant. The natural death rates of human and sand-fly are denoted by $\mu_{H}$ and $\mu_{V}$. Now, susceptible human become infected through mass action after interacted with the infected vector, where $\beta$ is the per capita biting rate of vector on human and $\pi$ is the transmission probability per bite per human (Bacaer and Guernaoui, 2006). We have assumed that $P(t)=S_{H}(t)+I_{H}(t)$ is a constant and $V(t)=S_{V}(t)+i_{V}(t)$, where the first component of the vector population is the number of pathogen-free (susceptible) vectors $\left(s_{V}(t)\right)$ and $i_{V}(t)$ is the number of vectors, who carries the pathogen at time $t$. The pathogen can be transmitted from parent (female) to offspring in the vector population. So it is assumed that all newborn vectors are susceptible and vertical transmission can be neglected. Also the susceptible vectors become infected after biting infected human at a rate $\beta$ with transmission probability $\hat{\pi}$ per bite from human to sand-fly, so that the incidence of newly infected vectors is given by a mass action term $\beta \hat{\pi} s_{V}(t) I_{H}(t)$. In contrast to the host population, once the vectors become carriers of 
International Journal of Mathematical, Engineering and Management Sciences

Vol. 1, No. 3, 139-149, 2016

https://dx.doi.org/10.33889/IJMEMS.2016.1.3-015

the micro parasite, they carry it for life (Yang et al., 2010). Based on the above assumptions, the system that describes the disease dynamics is given by:

$\dot{s}_{V}=\lambda_{V}-\beta \hat{\pi} s_{V}(t) \frac{I_{H}(t)}{P(t)}-\mu_{V} s_{V}(t)$,

$i_{V}=\beta \hat{\pi} s_{V}(t) \frac{I_{H}(t)}{P(t)}-\mu_{V} i_{V}(t)$.

It can be easily seen that the vector population is constant, i.e., $\lim _{\mathrm{t} \rightarrow \infty} \frac{\lambda_{V}}{\mu_{V}}$ for all $t>0$ provided that $s_{V}(0)+i_{V}(0)=\frac{\lambda_{V}}{\mu_{V}}$. The disease dynamics of human and sand-fly population are described in Fig. 1.

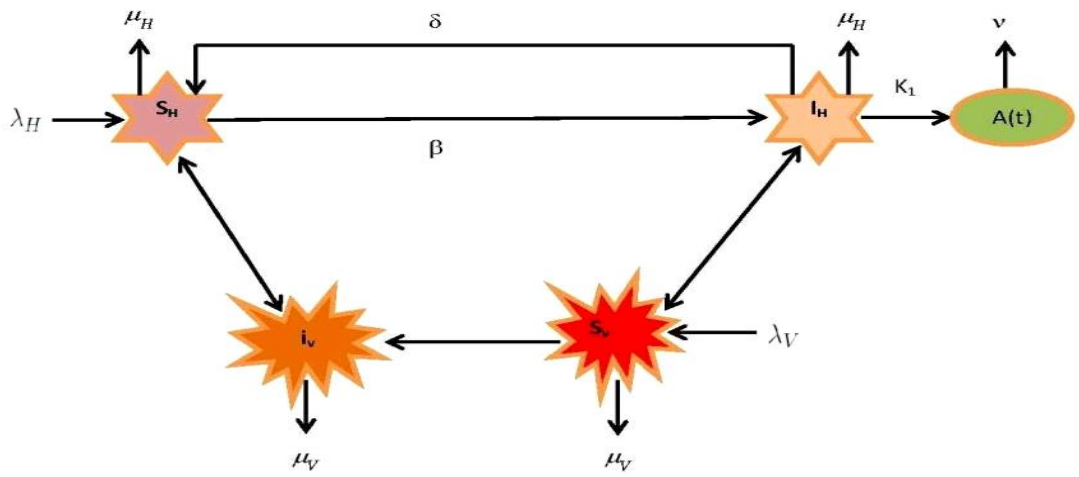

Fig. 1. Compartmental diagram of the model system (2)

We also assume that $A(t)$ is the aware class. The natural recovery rate from infected human to susceptible human class is $\delta$, the reduction rate of infected human population due to awareness is denoted as $k_{l}$. The growth rate of aware population is $k_{2}$, the reduction rate of awareness programs due to incompetence is $v$. The rate of awareness programs is assumed to be proportional to the number of infected individuals. It is considered that due to the awareness programs, susceptible individuals form a different class and avoid contact with the infected human. It is also considered that $k_{2} \gg v$. From above diagram, we have obtained the following complete system of equations given below:

$$
\begin{aligned}
& \dot{S}_{H}=\lambda_{V}-\beta \pi i_{V}(t) \frac{S_{H}(t)}{P(t)}-\mu_{H} S_{H}(t)+\delta I_{H}(t), \\
& \dot{I}_{H}=\beta \pi i_{V}(t) \frac{S_{H}(t)}{P(t)}-\mu_{H} I_{H}(t)-\delta I_{H}(t)-k_{1} A(t) I_{H}(t), \\
& \dot{l_{V}}=\beta \hat{\pi}\left(\frac{\lambda_{V}}{\mu_{V}}-i_{V}(t)\right) \frac{I_{H}(t)}{P(t)}-\mu_{V} i_{V}(t), \\
& \dot{A}=k_{2} I_{H}(t)-v A(t) .
\end{aligned}
$$

We have established that the system is bounded for the conditions $0<P(t) \leq \frac{\lambda_{H}}{\mu_{H}}, V(t) \leq \frac{\lambda_{V}}{\mu_{V}}$ and $A(t) \leq \sqrt{\frac{k_{2} \lambda_{H}}{k_{1} v}}$ as $t \rightarrow \infty$. 
International Journal of Mathematical, Engineering and Management Sciences

Vol. 1, No. 3, 139-149, 2016

https://dx.doi.org/10.33889/IJMEMS.2016.1.3-015

\section{Theoretical Analysis of the System}

\subsection{Existence Condition}

In this system, there are two equilibrium points such as disease-free equilibrium $E_{0}\left(\bar{S}_{H}, 0,0,0\right) \equiv$ $E_{0}\left(\frac{\lambda_{H}}{\mu_{H}}, 0,0,0\right)$ and another is endemic equilibrium $E^{*}\left(S_{H}^{*}, I_{H}^{*}, i_{V}^{*}, A^{*}\right)$, where $S_{H}^{*}=$ $\frac{-k_{1} k_{2} I_{H}^{*}{ }^{2}-\mu_{H} v I_{H}^{*}+\lambda_{H} v}{\mu_{H} v}, i_{V}^{*}=\frac{\frac{\beta \hat{\pi} \lambda_{V}}{P \mu_{V}} I_{H}^{*}}{\mu_{V}+\frac{\beta \hat{\pi}}{P} I_{H}^{*}}, A^{*}=\frac{k_{2} I_{H}^{*}}{v}$ and $I_{H}^{*}$ is determined from the equation $a I_{H}^{* 2}+$ $b I_{H}^{*}+c=0$, where $a=\frac{\beta \widehat{\pi} k_{1} k_{2}}{P v}, b=\left(\frac{\mu_{V} k_{1} k_{2}}{v}+\frac{\beta \hat{\pi} \mu_{H}}{P}+\frac{\delta \beta \widehat{\pi}}{P}\right)$ and $c=\mu_{V} \mu_{H}+\delta \mu_{V}-\frac{\beta^{2} \pi \widehat{\pi} \lambda_{V}}{P^{2} \mu_{V}}$. Now, the endemic equilibrium $E^{*}$ exists if $\beta>P \mu_{V} \sqrt{\frac{\left(\mu_{H}+\delta\right)}{\lambda_{V} \pi \hat{\pi}}}$.

Biological Interpretation: If the biting rate is increased and exceeds its critical value, then the system moves to the endemic state and disease persists. For an increased value of biting rate, the level of infected human population is increased with a fixed transmission rate. Simultaneously, with an increased value of transmission rate, the infected human population is enhanced more than the susceptible human population for a preferred biting rate.

\subsection{Stability of the System}

The Jacobian matrix for the model system (2) at the disease-free equilibrium is given by,

$$
\left.j\right|_{\left(\bar{S}_{H}, 0,0,0\right)}=\left(\begin{array}{ccrr}
-\mu_{H} & \delta & -\frac{\beta \pi \bar{S}_{H}}{P} & 0 \\
0 & -\mu_{H}-\delta & \frac{\beta \pi \bar{S}_{H}}{P} & 0 \\
0 & \frac{\beta \hat{\pi} \lambda_{V}}{P \mu_{V}} & -\mu_{V} & 0 \\
0 & k_{2} & 0 & -v
\end{array}\right) .
$$

The characteristic equation for the disease-free equilibrium $E_{0}$ is stated as

$$
\lambda^{2}+\left(\mu_{H}+\mu_{V}+\delta\right) \lambda+\left(\mu_{H}+\delta\right) \mu_{V}-\frac{\beta^{2} \pi \hat{\pi} \lambda_{H} \lambda_{V}}{P^{2} \mu_{H} \mu_{V}}=0
$$

The threshold condition is determined by the sign of constant term. It follows that the basic reproduction number $R_{0}=\frac{\beta^{2} \pi \widehat{\pi} \mu_{H} \lambda_{V}}{\lambda_{H} \mu_{V}^{2}\left(\mu_{H}+\delta\right)}$. Thus if $R_{0}<1$, then the disease-free equilibrium is stable, while if $R_{0}>1$, then the disease-free equilibrium is unstable and the system moves to its endemic state.

Finally the Jacobian matrix at the endemic equilibrium $E^{*}\left(S_{H}^{*}, I_{H}^{*}, i_{V}^{*}, A^{*}\right)$ is furnished by, 
International Journal of Mathematical, Engineering and Management Sciences

Vol. 1, No. 3, 139-149, 2016

https://dx.doi.org/10.33889/IJMEMS.2016.1.3-015

$$
\left.j\right|_{\left(S_{H}^{*}, I_{H}^{*}, i_{V}^{*}, A^{*}\right)}=\left[\begin{array}{cccc}
-\frac{\beta \pi i_{V}^{*}}{P}-\mu_{H} & \delta & -\frac{\beta \pi S_{H}^{*}}{P} & 0 \\
\frac{\beta \pi i_{V}^{*}}{P} & -\mu_{H}-\delta-k_{1} A^{*} & \frac{\beta \pi S_{H}^{*}}{P} & -k_{1} I_{H}^{*} \\
0 & \frac{\beta \pi}{P}\left(\frac{\lambda_{V}}{\mu_{V}}-i_{V}^{*}\right) & -\frac{\beta \pi I_{H}^{*}}{P}-\mu_{V} & 0 \\
0 & k_{2} & 0 & -v
\end{array}\right] .
$$

The characteristic equation is given by,

$$
\lambda^{4}+a_{1} \lambda^{3}+a_{2} \lambda^{2}+a_{3} \lambda+a_{4}=0
$$

where

$$
\begin{aligned}
& a_{1}=-\left(x_{11}+x_{22}+x_{33}+x_{44}\right) \text {, } \\
& a_{2}=x_{11} x_{22}+x_{11} x_{33}+x_{11} x_{44}+x_{22} x_{33}+x_{22} x_{44} \\
& +x_{33} x_{44}-x_{24} x_{42}-x_{12} x_{21}-x_{23} x_{32} \text {, } \\
& a_{3}=x_{11} x_{24} x_{42}+x_{12} x_{21} x_{44}+x_{24} x_{33} x_{42}+x_{12} x_{21} x_{33}+x_{11} x_{23} x_{32}+x_{23} x_{32} x_{44} \\
& -x_{11} x_{22} x_{33}-x_{11} x_{22} x_{44}-x_{22} x_{33} x_{44}-x_{13} x_{21} x_{32}-x_{11} x_{33} x_{44} \text {, } \\
& a_{4}=x_{11} x_{22} x_{33} x_{44}+x_{13} x_{21} x_{32} x_{44}-x_{11} x_{23} x_{32} x_{44}-x_{12} x_{21} x_{33} x_{44}-x_{11} x_{24} x_{33} x_{42} \text {, } \\
& x_{11}=-\frac{\beta \pi i_{V}^{*}}{P}-\mu_{H}, x_{12}=\delta, x_{13}=-\frac{\beta \pi S_{H}^{*}}{P}, x_{21}=\frac{\beta \pi i_{V}^{*}}{P}, x_{22}=-\mu_{H}-\delta-k_{1} A^{*}, x_{23}= \\
& \frac{\beta \pi S_{H}^{*}}{P}, x_{24}=-k_{1} I_{H}^{*}, x_{32}=\frac{\beta \widehat{\pi}}{P}\left(\frac{\lambda_{V}}{\mu_{V}}-i_{V}^{*}\right), x_{33}=-\frac{\beta \hat{\pi} I_{H}^{*}}{P}-\mu_{V}, x_{42}=k_{2} \text { and } x_{44}=-v \text {. }
\end{aligned}
$$

Hence the endemic equilibrium point $E^{*}\left(S_{H}^{*}, I_{H}^{*}, i_{V}^{*}, A^{*}\right)$ is said to be stable if $a_{1} a_{2}-a_{3}>0$ and $a_{1} a_{2} a_{3}-a_{1}^{2} a_{4}-a_{3}^{2}>0$.

\section{Awareness Programme through Impulsive Mode}

In this section, we wish to observe the impulsive effect of awareness campaigning in fixed time interval. It helps to avoid the contact between human and infected vector. Here we have chosen distinct time interval for campaigning. During the programme, the number of aware population in the environment is increased by some proportion $\rho$. So, infected human as well as vector population are reduced. The awareness campaigning does not have any fatal impact on the system dynamics as we have studied the model through impulsive mode. Thus impulsive differential equation takes the form:

$\frac{d(A(t))^{2}}{d t}=\xi-v^{\prime}(A(t))^{2}, t \neq t_{k}$

$$
\Delta A=\rho A, \quad t=t_{k},
$$

where

$$
\frac{2 k_{2} \lambda_{H}}{k_{1}}=\xi \text { (say) }
$$


International Journal of Mathematical, Engineering and Management Sciences

Vol. 1, No. 3, 139-149, 2016

https://dx.doi.org/10.33889/IJMEMS.2016.1.3-015

For single impulsive cycle $t_{k-1} \leq t \leq t_{k}$, the general solution is given by,

$A_{k+1}^{+}=\frac{\xi^{\prime}}{v^{\prime}}\left\{1-e^{-v^{\prime}\left(t_{k}-t_{k-1}\right)}\right\}+A_{k}^{-} e^{-v^{\prime}\left(t_{k}-t_{k-1}\right)}$, using $\frac{\xi^{\prime}}{v^{\prime}}=\sqrt{\frac{k_{2} \lambda_{H}}{k_{1} v}}$,

where $A\left(t_{k}^{+}\right)=A_{k}^{+}$and $A\left(t_{k}^{-}\right)=A_{k}^{-}$. Now,

$A_{1}^{+}=\frac{\xi^{\prime}}{v^{\prime}}, A_{1}^{-}=(1-\rho) \frac{\xi^{\prime}}{v^{\prime}}, A_{2}^{+}=(1-\rho) \frac{\xi^{\prime}}{v^{\prime}} e^{-v^{\prime}\left(t_{2}-t_{1}\right)}+\frac{\xi^{\prime}}{v^{\prime}}\left\{1-e^{-v^{\prime}\left(t_{2}-t_{1}\right)}\right\}$,

$A_{2}^{-}=(1-\rho) A_{2}^{+}=(1-\rho)^{2} \frac{\xi^{\prime}}{v^{\prime}} e^{-v^{\prime}\left(t_{2}-t_{1}\right)}+(1-\rho) \frac{\xi^{\prime}}{v^{\prime}}\left\{1-e^{-v^{\prime}\left(t_{2}-t_{1}\right)}\right\}$,

$A_{3}^{+}=\frac{\xi^{\prime}}{v^{\prime}}\left[(1-\rho)^{2} e^{-v^{\prime}\left(t_{3}-t_{1}\right)}+(1-\rho) e^{-v^{\prime}\left(t_{3}-t_{2}\right)}+1-(1-\rho) e^{-v^{\prime}\left(t_{3}-t_{1}\right)}-e^{-v^{\prime}\left(t_{3}-t_{2}\right)}\right]$,

$A_{4}^{+}=\frac{\xi^{\prime}}{v^{\prime}}\left[(1-\rho)^{3} e^{-v^{\prime}\left(t_{4}-t_{1}\right)}+(1-\rho)^{2} e^{-v^{\prime}\left(t_{4}-t_{2}\right)}+(1-\rho) e^{-v^{\prime}\left(t_{4}-t_{3}\right)}+1-(1-\right.$

$\left.\rho)^{2} e^{-v^{\prime}\left(t_{4}-t_{1}\right)}-(1-\rho) e^{-v^{\prime}\left(t_{4}-t_{2}\right)}-e^{-v^{\prime}\left(t_{4}-t_{3}\right)}\right]$.

Therefore the general solution becomes,

$$
\begin{aligned}
A_{n}^{+}=\frac{\xi^{\prime}}{v^{\prime}} & {\left[(1-\rho)^{(n-1)} e^{-v^{\prime}\left(t_{n}-t_{1}\right)}+(1-\rho)^{(n-2)} e^{-v^{\prime}\left(t_{n}-t_{2}\right)}+\cdots+(1-\rho) e^{-v^{\prime}\left(t_{n}-t_{n-1}\right)}\right.} \\
& \left.+1-(1-\rho)^{(n-2)} e^{-v^{\prime}\left(t_{n}-t_{1}\right)}-(1-\rho)^{(n-3)} e^{-v^{\prime}\left(t_{n}-t_{2}\right)}-\cdots-e^{-v^{\prime}\left(t_{n}-t_{n-1}\right)}\right] .
\end{aligned}
$$

This is the general solution for the maximum number of aware population present in the society due to awareness campaign mainly driven by social mass media.

\subsection{Awareness for Fixed Time Interval}

For fixed time interval, i.e., $\tau=t_{n}-t_{n-1}$ is constant, we have,

$$
\begin{aligned}
& A_{n}^{+}=\frac{\xi^{\prime}}{v^{\prime}}\left[1+(1-\rho) e^{-v^{\prime} \tau}+(1-\rho)^{2} e^{-2 v^{\prime} \tau}+\cdots+(1-\rho)^{n-1} e^{-v^{\prime}(n-1) \tau}-\right. \\
& e^{-v^{\prime} \tau}\left\{1+(1-\rho) e^{-v^{\prime} \tau}+\cdots+(1-\rho)^{(n-2)} e^{-v^{\prime}(n-2) \tau}\right]=\frac{\xi^{\prime}}{v^{\prime}}\left[\frac{1-(1-\rho)^{n} e^{-v^{\prime} n \tau}}{1-(1-\rho) e^{-v^{\prime} \tau}}-\right. \\
& \left.e^{-v^{\prime} \tau} \frac{1-(1-\rho)^{(n-1)} e^{-v^{\prime}(n-1) \tau}}{1-(1-\rho) e^{-v^{\prime} \tau}}\right] .
\end{aligned}
$$

Therefore,

$$
\lim _{\mathrm{n} \rightarrow \infty} A_{n}^{+}=\frac{\xi^{\prime}}{v^{\prime}}\left[\frac{1}{1-(1-\rho) e^{-v^{\prime} \tau}}-e^{-v^{\prime} \tau} \frac{1}{1-(1-\rho) e^{-v^{\prime} \tau}}\right]=\frac{\xi^{\prime}}{v^{\prime}}\left[\frac{1-e^{-v^{\prime} \tau}}{1-(1-\rho) e^{-v^{\prime} \tau}}\right]
$$

This is maximum number of human present in the environment that have to aware. To keep this under the threshold value $\widetilde{A}$ we have,

$$
\frac{\xi^{\prime}}{v^{\prime}}\left[\frac{1-e^{-v^{\prime} \tau}}{1-(1-\rho) e^{-v^{\prime} \tau}}\right]<\widetilde{A},
$$

which implies

$$
\begin{gathered}
\tau<\frac{1}{v^{\prime}} \ln \left[\frac{\xi^{\prime}-\widetilde{A} v^{\prime}(1-\rho)}{\xi^{\prime}-\widetilde{A} v^{\prime}}\right]=\tau_{\max }(\rho) \text { (say). } \\
\text { i.e., } \tau<\frac{1}{2 v} \ln \left[\frac{\sqrt{\frac{k_{2} \lambda_{H}}{k_{1} v}}-\widetilde{A} 2 v(1-\rho)}{\sqrt{\frac{k_{2} \lambda_{H}}{k_{1} v}}-\widetilde{A} 2 v}\right]=\tau_{\max }(\rho) \text { (say). }
\end{gathered}
$$


International Journal of Mathematical, Engineering and Management Sciences

Vol. 1, No. 3, 139-149, 2016

https://dx.doi.org/10.33889/IJMEMS.2016.1.3-015

Biological Interpretation: If the time interval $\tau$ be less than some predetermined quantity $\left(\tau_{\max }\right)$, then we can reduce the number of infected human population through awareness campaign under the threshold value $\widetilde{A}$ in a real-life scenario.

\section{Numerical Simulation}

\begin{tabular}{|c|c|c|}
\hline Parameter & Range & $\begin{array}{c}\text { Default Value } \\
\left(\mathbf{d a y}^{-1} \text { ) }\right.\end{array}$ \\
\hline$\lambda_{H}$ & $300-318$ & 317 \\
\hline$\Lambda_{V}$ & $14950-15000$ & 14950 \\
\hline$\mu_{H}$ & $0.3-0.4$ & 0.3 \\
\hline$\mu_{V}$ & $0.189-0.195$ & 0.189 \\
\hline$\beta$ & $0.21-0.29$ & 0.25 \\
\hline$\pi$ & $0.22-0.3$ & 0.3 \\
\hline$\hat{\pi}$ & $0.071428-0.1$ & 0.1 \\
\hline$K_{I}$ & $0.0284-0.0324$ & 0.03 \\
\hline$K_{2}$ & $0.0378-0.401$ & 0.04 \\
\hline$v$ & $0.0028-0.00034$ & 0.003 \\
\hline$\delta$ & $0.00281-0.042$ & 0.03 \\
\hline$\rho$ & $2-9$ & 2,8 \\
\hline
\end{tabular}

Table 1. List of parameters used in the model equation (2)

Note. The recruitment rate of human population is taken as $\lambda_{H}$, the recruitment rate of sand-fly population is considered as $\lambda_{V}$, the natural death rate of sand-fly is denoted $\mu_{V}, \beta$ is the per capita biting rate of vector on human. All the above mentioned parameter values are taken from Biswas et al. (2014) and ELmojtaba et al. (2010); the numerical values of transmission probability per bite per human $\pi$ and the transmission probability $\hat{\pi}$ per bite from human to sand-fly are taken from Bathena (2009) and ELmojtaba et al. (2010); the reduction rate of awareness programs due to incompetence $(v)$ is taken from Misra et al. (2011); the natural death rate of human $\mu_{H}$, the reduction rate of infected human population due to awareness $k_{1}$ and the growth rate of aware population $k_{2}$ are estimated; the natural recovery rate from infected human to susceptible human class $\delta$ and the number of aware population in the environment, increased by some proportion $\rho$ are also estimated.

In this section, we have analyzed the mathematical model (2) through numerical simulation. The values of the model parameters, used in our numerical simulation, are given in Table 1. From Fig. 2 , it is clear that when biting rate is below its threshold value $(\beta<0.25)$, the system attains its disease-free state. But if the biting rate crosses its threshold value $(\beta>0.25)$, the system moves to its endemic state and the disease persists (see Fig. 3). The effects of awareness campaign are exhibited in Fig. 4 and Fig. 6. In Fig. 6, we have considered $\rho=2$ and $\rho=8$ with $\tau=2$. It is clearly observed that for awareness campaign with twice a day in a two days interval, the infected system moves to infection-free state. Also if we increase the rate of campaign in a day, the system reaches towards better situation. If the awareness campaign is made in twice a day, the system achieves better result after 6 days or more than that. But increasing the effectiveness of campaign 8 times in a day, the system gives much more enhanced results (i.e., number of susceptible human increases more quickly). 
International Journal of Mathematical, Engineering and Management Sciences

Vol. 1, No. 3, 139-149, 2016

https://dx.doi.org/10.33889/IJMEMS.2016.1.3-015
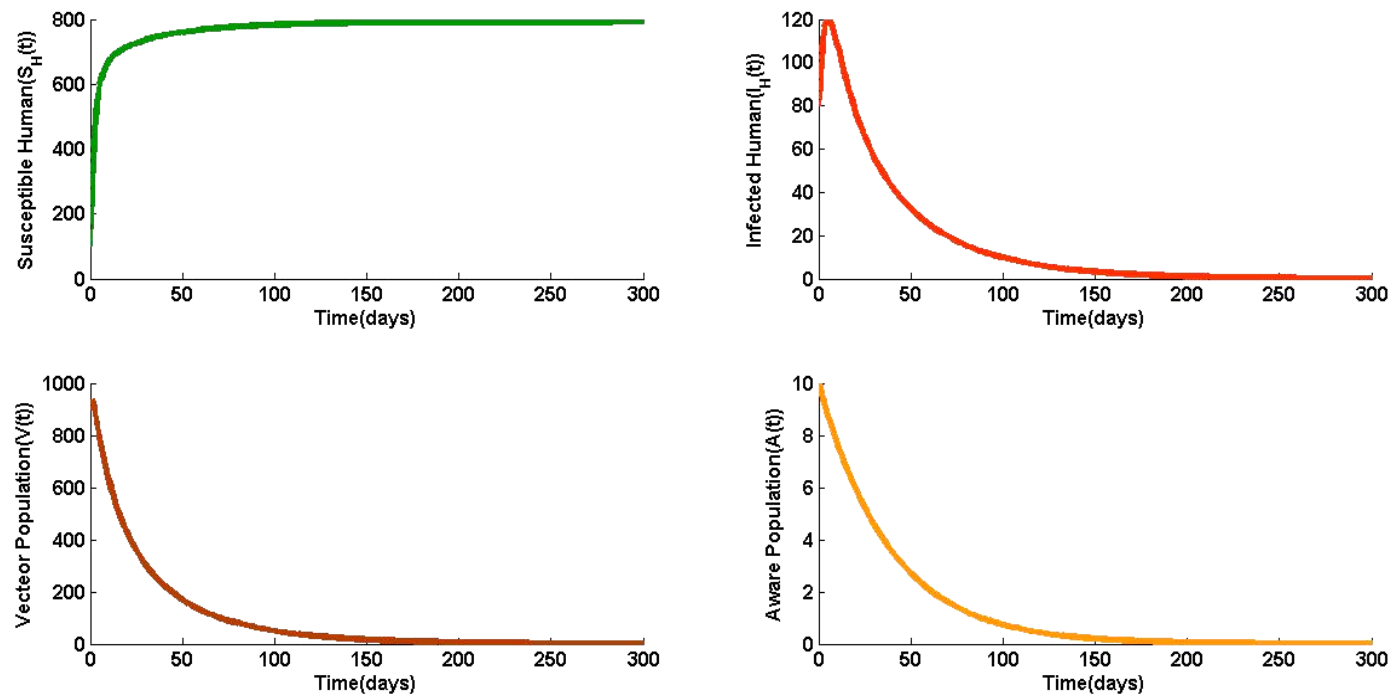

Fig. 2. Behavior of different population for $\mathrm{R}_{0}<1$, when the system attains its disease-free state
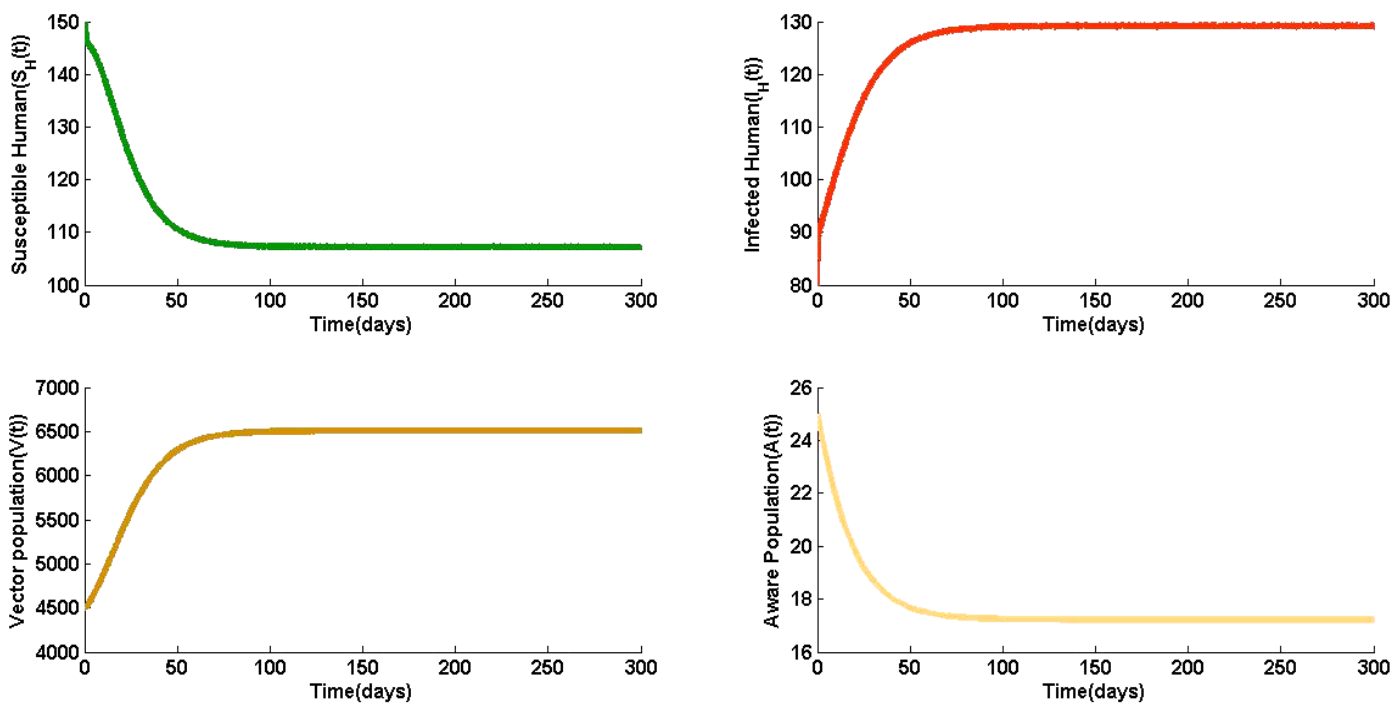

Fig. 3. Behavior of different population for $\mathrm{R}_{0}>1$, when the system attains its endemic state

Thus effectiveness of the awareness campaign provides a superior effect moving the system towards its disease-free situation. In Fig. 4 and Fig. 5, we have observed that infected human population can be controlled but from Fig. 6, it is very clear that infected human population goes to lower level by choosing proper campaigning frequency and interval. It is not possible to choose frequency in only awareness campaign without impulsive mode. It only considers the rate of awareness campaign in a fixed manner in respect to infected human. So that awareness campaign through impulsive way provides better result than normal campaign. 
International Journal of Mathematical, Engineering and Management Sciences

Vol. 1, No. 3, 139-149, 2016

https://dx.doi.org/10.33889/IJMEMS.2016.1.3-015
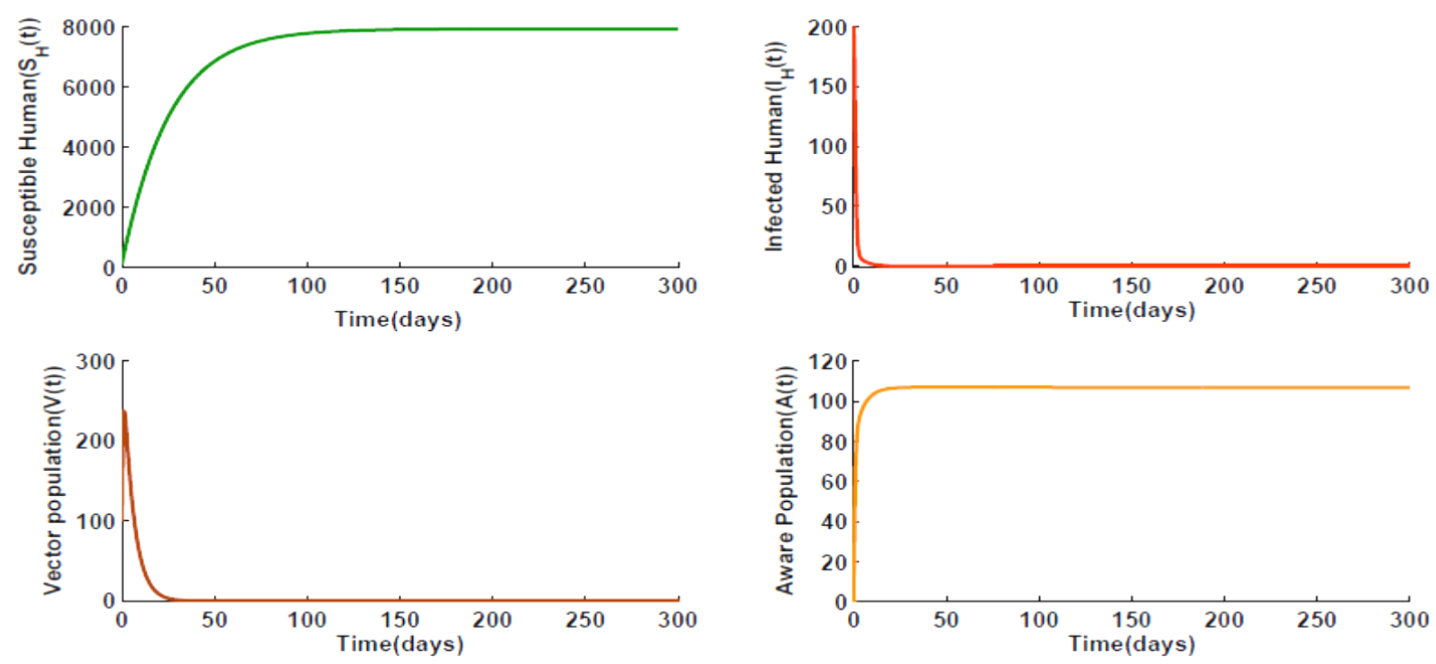

Fig. 4. Population density as a function of time when awareness campaigning is circulated by media
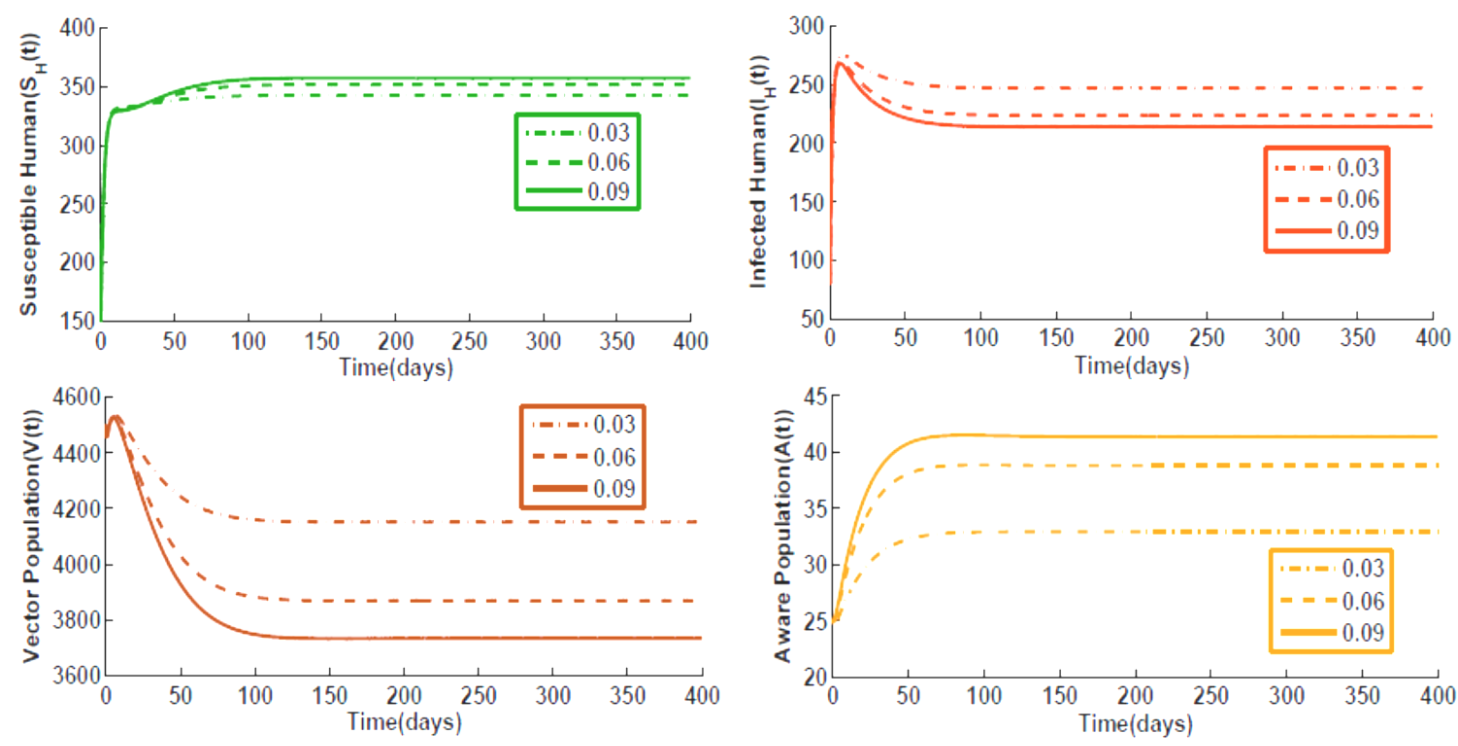

Fig. 5. Density of four populations for different values of $k_{2}$

\section{Discussion}

It is important to know the contact process between human and infected vector. If it is possible to manage the contact process to a minimum, then extermination of the disease can be achieved. In this connection, awareness campaign can perform a momentous responsibility for controlling the disease. We have investigated the system in absence of awareness programmes. The disease-free situation exists, if $\mathrm{R}_{0}<1$ and if $\mathrm{R}_{0}>1$, the disease-free state drops its stability and the system tends towards the endemic situation. Susceptible host population is sharply decreased up to 45 days (approx.) and infected host population is gradually increased up to more or less 50 days, when the 
International Journal of Mathematical, Engineering and Management Sciences

Vol. 1, No. 3, 139-149, 2016

https://dx.doi.org/10.33889/IJMEMS.2016.1.3-015

disease exists. Moreover, the biting rate of sand-fly is considered more important for spread of the disease. Likewise, growth rate of aware population is very significant parameter under mathematical perspective. The change in the behavioral structure of the system depends efficiently on biting rate of sand-fly and growth rate of aware population. So if we are able to control the interaction between human and sand-fly by awareness campaign periodically, then disease can automatically be restrained.

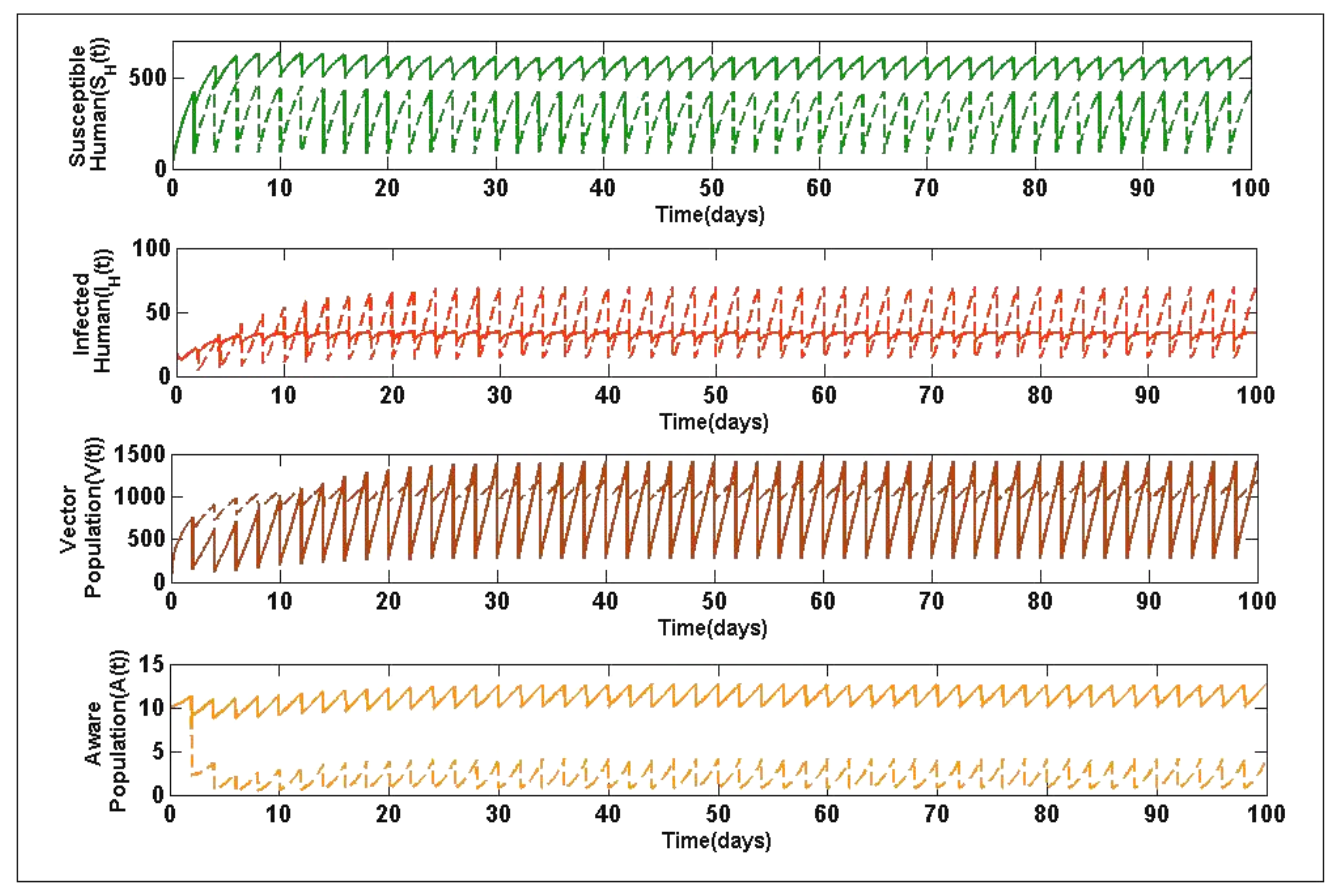

Fig. 6. The impulsive behavior of the model system with awareness campaign after two days interval with $\rho$ $=2$ (“- - -") and $\rho=8$ (“-”")

\section{Conclusion}

In presence of effective awareness programme implemented impulsively the abolition of disease transmission is made possible in due time. The disease can be controlled by awareness programs mainly driven by social mass media with proper selection of the frequency and interval of campaigning. Complete termination of cutaneous leishmaniasis is difficult in realistic situation because the disease has different vectors and reservoirs. According to the evidences obtained from the analytical and numerical findings our model based approach suggests that awareness campaign implemented in impulsive mode is able to gain new insights extensively to fight against cutaneous leishmaniasis.

\section{References}

Bacaër, N., \& Guernaoui, S. (2006). The epidemic threshold of vector-borne diseases with seasonality. Journal of Mathematical Biology, 53(3), 421-436.

Bathena, K. (2009). A Mathematical model of cutaneous leishmaniasis. Thesis. Rochester Institute of Technology.

Biswas, D., Kesh, D. K., Datta, A., Chatterjee, A. N., \& Roy, P. K. (2014). A mathematical approach to 
International Journal of Mathematical, Engineering and Management Sciences

Vol. 1, No. 3, 139-149, 2016

https://dx.doi.org/10.33889/IJMEMS.2016.1.3-015

control cutaneous leishmaniasis through insecticide spraying. Sop Transactions on Applied Mathematics, 1(2), 44-54.

Biswas, D., Roy, P. K., Li, X. Z., Basir, F. A., \& Pal, J. (2016). Role of macrophage in the disease dynamics of cutaneous Leishmaniasis: a delay induced mathematical study. Communications in Mathematical Biology and Neuroscience, 2016(4), pp. 1-31.

Chaves, L. F., \& Hernandez, M. J. (2004). Mathematical modelling of American cutaneous leishmaniasis: incidental hosts and threshold conditions for infection persistence. Acta Tropica, 92(3), 245-252.

Das, P., Mukherjee, D., \& Sarkar, A. K. (2007). Effect of delay on the model of American cutaneous leishmaniasis. Journal of Biological Systems, 15(02), 139-147.

ELmojtaba, I. M., Mugisha, J. Y. T., \& Hashim, M. H. (2010). Mathematical analysis of the dynamics of visceral leishmaniasis in the Sudan. Applied Mathematics and Computation, 217(6), 2567-2578.

Funk, S., Gilad, E., Watkins, C., \& Jansen, V. A. (2009). The spread of awareness and its impact on epidemic outbreaks. Proceedings of the National Academy of Sciences, 106(16), 6872-6877.

Killick-Kendrick, R. (1999). The biology and control of phlebotomine sand flies. Clinics in Dermatology, 17(3), 279-289.

Länger, B. M., Pou-Barreto, C., González-Alcón, C., Valladares, B., Wimmer, B., \& Torres, N. V. (2012). Modeling of leishmaniasis infection dynamics: novel application to the design of effective therapies. BMC Systems Biology, 6(1), 1.

Liu, Y. \& Cui, J. (2008). The impact of media convergence on the dynamics of infectious diseases, International Journal of Biomathematics, 1, 65-74.

Lysenko, A. J., Beljaev, A. E., Peters, W., \& Killick-Kendrick, R. (1987). Quantitative approaches to epidemiology. The leishmaniases in biology and medicine. Volume I. Biology and epidemiology, 263290.

Marcos, C. D. \& Moreira, H. N. (2007). A mathematical model of immune response in cutaneous leishmaniasis. Journal of Biological Systems, 15(3), pp. 313 - 354

Misra, A. K., Sharma, A., \& Shukla, J. B. (2011). Modeling and analysis of effects of awareness programs by media on the spread of infectious diseases. Mathematical and Computer Modelling, 53(5), 12211228.

Misra, A. K., Sharma, A., \& Singh, V. (2011). Effect of awareness programs in controlling the prevalence of an epidemic with time delay. Journal of Biological Systems, 19(02), 389-402.

Nyabadza, F., Chiyaka, C., Mukandavire, Z., \& Hove-Musekwa, S. D. (2010). Analysis of an HIV/AIDS model with public-health information campaigns and individual withdrawal. Journal of Biological Systems, 18(2), 357-375.

Rabinovich, J. E., \& Feliciangeli, M. D. (2004). Parameters of leishmania braziliensis transmission by indoor Lutzomyia ovallesi in Venezuela. The American Journal of Tropical Medicine and Hygiene, 70(4), 373382.

Reithinger, R., Dujardin, J. C., Louzir, H., Pirmez, C., Alexander, B., \& Brooker, S. (2007). Cutaneous leishmaniasis. The Lancet Infectious Diseases, 7(9), 581-596.

World Health Organization. (2009). Leishmaniasis: magnitude of the problem. World Health Organization, Geneva.

Yang, H., Wei, H., \& Li, X. (2010). Global stability of an epidemic model for vector-borne disease. Journal of Systems Science and Complexity, 23(2), 279-292. 